\title{
Caracterização da composição e estrutura da fauna epígea em monocultivos de café e eucalipto, no Norte do Espírito Santo
}

\author{
Characterization of the composition and structure of the epigeal fauna in coffee and eucalyptus \\ monocultures, in the North of Espírito Santo \\ Caracterización de la composición y estructura de la fauna epigea en monocultivos de café y \\ eucalipto, en el Norte de Espírito Santo
}

\section{Resumo}

O objetivo deste estudo foi caracterizar e comparar a abundância e diversidade da fauna epígea entre diferentes sistemas agrícolas, no município de Sooretama, ES. Foram selecionadas duas áreas a saber: monocultivo de café (Coffea conephora) e monocultivo de eucalipto (Eucalyptus citriodora), ambas com 5 anos. A amostragem da fauna epígea foi realizada com o auxílio de armadilhas de queda do tipo pitfall traps. Em cada área, foram instaladas 10 armadilhas, que permaneceram no campo por 7 dias. A partir da identificação e quantificação dos organismos, foram calculados o número de indivíduos por armadilha ao dia, riqueza média e riqueza total, e os índices de diversidade de Shannon, uniformidade de Pielou e dominância de Simpson. Os grupos taxonômicos foram ainda classificados quanto à funcionalidade. No plantio de café, o grupo com maior atividade foi Isopoda, com 52,41\% de indivíduos coletados, seguido pelo grupo Formicidae com $18,46 \%$. Na área de plantio de eucalipto o grupo taxonômico de maior atividade foi o Formicidae com $83,34 \%$ de indivíduos coletados. O plantio de café favoreceu a atividade e diversidade da fauna, sendo verificada a maior abundância de indivíduos, riqueza de grupos e maior diversidade nessa área. A análise da funcionalidade mostrou que o plantio de café promoveu melhores condições para o grupo saprófago Isopoda e o plantio de eucalipto o grupo social (Formicidae).

Palavras-chave: Cultivos agrícolas; Fauna epígea; Atividade; Diversidade; Grupos funcionais.

\begin{abstract}
The aim of this study was to characterize and compare the abundance and diversity of epigeal fauna between different agricultural systems, in the municipality of Sooretama, ES. Two areas were selected: monoculture of coffee (Coffea conephora) and eucalyptus (Eucalyptus citriodora), both with 5 years old. The sampling of the epigeal fauna was carried out using pittfall traps. In each area, were installed 10 traps, which remained in the field for 7 days. After identification and quantification, were determined the number of organisms per traps per day, the total and average richness, and the Shannon diversity, Pielou uniformity and Simpson dominance indexes. The taxonomic groups also were classified according to functionality. In the coffee monoculture, the group with the highest activity was Isopoda $(52.41 \%)$, followed by the Formicidae groupd $(18.46 \%)$. In the eucalyptus plantation, the taxonomic group with the highest activity was Formicidae with $83.34 \%$. The planting of coffee favoured the activity and diversity of the epigean fauna, with the higher individual's abundance, richness, and diversity than the eucalyptus monoculture. The analysis of the functionality showed that the coffee monoculture promoted better conditions for activity of the Saprophagous group (Isopoda) and the eucalyptus planting for the Social group (Formicidae).
\end{abstract}

Keywords: Agricultural crops; Epigean fauna; Activity; Diversity; Functional groups.

\section{Resumen}

El objetivo de este estudio fue caracterizar y comparar la abundancia y diversidad de fauna epigea entre diferentes sistemas agrícolas en el municipio de Sooretama, ES. Fueron seleccionadas dos áreas: monocultivo de café (Coffea 
conephora) y monocultivo de eucalipto (Eucalyptus citriodora), ambas con 5 años. El muestreo de la fauna epigea se realizó con la ayuda de trampas de caída del tipo pitfall traps. En cada área se instalaron 10 trampas, que permanecieron en el campo durante 7 días. A partir de la identificación y cuantificación de los organismos, se calculó el número de individuos por trampa por día, la riqueza media y total y los índices de diversidad de Shannon, uniformidad de Pielou y dominancia de Simpson, además los grupos taxonómicos se clasificaron según la funcionalidad. En la plantación de café, el grupo con mayor actividad fue Isopoda, con 52,41 \% de los individuos recolectados, seguido por el grupo Formicidae con 18,46 \%. En el área de plantación de eucalipto, el grupo taxonómico con mayor actividad fue Formicidae con $83,34 \%$ de individuos colectados. La siembra de café favoreció la actividad y diversidad de fauna, observándose la mayor cantidad de individuos, riqueza de grupos y mayor diversidad en esta área. El análisis de la funcionalidad mostró que la plantación de café promueve las mejores condiciones para el grupo saprófago Isopoda y la plantación de eucalipto al grupo social (Formicidae).

Palabras clave: Cultivos agrícolas; Fauna epigea; Actividad; Diversidad; Grupos funcionales.

\section{Introdução}

O solo é resultado de complexos processos e interações no tempo e no espaço, além de ser um reservatório chave da biodiversidade global, que é composta por microrganismos, flora e fauna (FAO, 2015). A biodiversidade do solo representa um componente imprescindível para o equilíbrio e a sustentabilidade do ambiente, e pode influenciar diversas funções e serviços ecossistêmcos. No que concerne à fauna invertebrada do solo, a biodiversidade pode ser compreendida como a variedade e variabilidade de espécies de organismos presentes no solo (Baretta et al., 2011), os quais são comumente classificados de acordo como o tamanho corporal, em: microfauna $(0,2 \mathrm{~mm})$, mesofauna $(0,2-2,0 \mathrm{~mm})$ e macrofauna $(>2,0 \mathrm{~mm})$ (Swift et al., 1979).

A serapilheira constitui um importante componente na manutenção da produtividade de ecossistemas florestais (Santos Neto et al., 2015) e juntamente com os componentes da vegetação influenciam a atividade e a diversidade de organismos que habitam o ambiente do solo (Oliveira et al., 2019). Considerando que a serapilheira consiste no habitat para os invertebrados que compõem a fauna do solo (Pereira et al., 2013), além de esse material ser a fonte de alimento para muitos desses organismos, sua decomposição é regulada pela qualidade do material que a constitui, bem como pela atividade dos organismos do solo e pelas condições físicas do ambiente (Oliveira et al., 2020).

A diversidade da fauna do solo está relacionada com uma grande variedade de recursos e micro-habitats que o sistema serapilheira-solo oferece (Lavelle et al., 1994), e esses organismos exercem importante papel no solo por realizarem diversas atividades. Ao ingerirem ou fragmentarem a serapilheira, aumentam a superfície desse material, para posterior ação dos microrganismos decompositores, promovendo a aceleração da decomposição, assim como, a incorporação desses resíduos no solo, processo que acelera a decomposição, e a distribuição da matéria orgânica no perfil do solo (Brown et al., 2015; Frouz, 2018). Assim, esses organismos participam de diversos serviços ambientais e estão diretamente relacionados à sustentabilidade dos ecossistemas.

Os organismos da fauna edáfica são sensíveis às mudanças ocorridas no ambiente do solo, decorrentes de práticas de cultivo e manejo, e das alterações no aporte de recursos vegetais e no microclima (Baretta et al., 2011; Brito et al., 2016; Casaril et al., 2019). Diante disso, as populações e a diversidade da fauna podem ser utilizadas como bioindicadores do uso do solo ou da sua fertilidade (Vaz-de-Melo et al., 2009), considerando sua rápida resposta às alterações no ambiente do solo (Casaril et al., 2019). Da mesma forma, os grupos funcionais da fauna têm respondido às mudanças no ambiente, frente ao manejo empregado (Marasas et al., 2001)

A partir do exposto, o objetivo deste estudo foi caracterizar e comparar a abundância e diversidade da fauna epígea entre áreas de monocultivo de café e eucalipto, no município de Sooretama, ES. 


\section{Metodologia}

\subsection{Caracterização das áreas de estudo}

O estudo foi realizado no Sítio Feitosa, Município de Sooretama, ES. A região está localizada entre os paralelos $18^{\circ}$ $53^{\prime} 53^{\prime \prime} \mathrm{S}$ e $19^{\circ} 30^{\prime} 00^{\prime \prime} \mathrm{S}$ e os meridianos $39^{\circ} 50^{\prime \prime} 00^{\prime} \mathrm{W}$ e $40^{\circ} 15^{\prime \prime} 50^{\prime} \mathrm{W}$ Gr, ao norte do estado do Espírito Santo. O clima da região é tropical úmido, com inverno seco e chuvas concentradas no verão, classificado como Aw de Köppen. A temperatura média anual é de $23^{\circ} \mathrm{C}$, sendo a média do mês mais quente de $25,6^{\circ} \mathrm{C}$, em fevereiro, e a média do mês mais frio $19,9^{\circ} \mathrm{C}$, em julho. O solo da área foi classificado como Latossolo Amarelo (Sá et al., 2003).

O presente estudo consiste numa pesquisa de campo, sendo um estudo de caso, de abordagem quantitativa. $\mathrm{O}$ suporte metodológico são todos os trabalhos feitos com fauna do solo usando o método de pitfall traps.

Foram selecionadas duas áreas a saber: uma área de plantio de café (Coffea conephora) e outra de eucalipto (Eucalyptus citriodora), ambas com mesmo tempo de instalação das culturas (5 anos). A área de café, desde a implantação, é adubada anualmente (2500 $\mathrm{kg} \mathrm{ha}^{-1}$ ano $^{-1}$ (NPK - 25-05-20) e submetida à desbrota e poda. A área de eucalipto encontrava-se na segunda rotação em talhadia, em que os indivíduos apresentaram altura média aproximada de 20 metros. Em ambas as áreas o material proveniente da desbrota, poda e desrama é depositado sobre o solo.

\subsection{Amostragem da fauna epígea}

Para a coleta da fauna invertebrada, foram utilizadas armadilhas do tipo "pitfall traps" de acordo com Moldenke (1994) e adaptado por Aquino et al. (2006). Em cada área foi delimitada uma parcela de 50 x 50 m, e nessas foram distribuídas aleatoriamente 10 armadilhas, que permaneceram no campo por um período de 7 dias. As armadilhas consistiram em recipientes plásticos com $10 \mathrm{~cm}$ de diâmetro e $10 \mathrm{~cm}$ de altura, as quais foram enterradas na interface solo-serrapilheira até que sua abertura ficasse no nível do solo. Foram ainda instaladas coberturas sobre as armadilhas, com o intuito de protegê-las da ocorrência de chuvas. Para tanto, utilizaram-se pratos plásticos com $15 \mathrm{~cm}$ de diâmetro, fixado no solo com auxílio de palitos de madeira. Em cada pote, foi depositada uma solução de ácido acetilsalicílico para preservação da fauna.

No laboratório, as amostras foram limpas e triadas com auxílio de lupa binocular, e os organismos da meso e da macrofauna foram quantificados e separados em grandes grupos taxonômicos (ordem e famílias) de acordo com Gallo et al. (2002).

\subsection{Análise de dados}

A partir da quantificação dos indivíduos de cada área, foram calculados as médias e o número de indivíduos por armadilha ao dia (Ind. $\mathrm{arm}^{-1}$. dia ${ }^{-1}$ ), considerando o tempo que permaneceram no campo, o que indica a atividade dos grupos. Adicionalmente, foram calculadas a riqueza total e riqueza média de grupos taxonômicos, e os índices de dominância de Simpson dada pela relação (Is = 1-L), sendo $\mathrm{L}=\mathrm{a}$ ni $(\mathrm{n}-1) / \mathrm{N}(\mathrm{N}-1)$; $\mathrm{ni}$ = número indivíduos do grupo "i”, $\mathrm{N}=$ (somatório da densidade de todos os grupos), diversidade de Shannon $\left(\mathrm{H}={ }^{\mathrm{R}} \Sigma \mathrm{i}_{\mathrm{i}=1} P_{i} \log _{2} P i\right.$, onde $P i$ é a proporção do grupo $i$ no total da amostra) e uniformidade de Pielou, o qual a equação deriva do índice de Shannon e é calculada a partir de: $U=(H / \ln R)$ (Odun 1986).

Os grupos também foram classificados quanto à funcionalidade em: Social (Formicidae), Social (Isoptera), Holometabolos, Saprófagos, Predadores, Micrófagos e Larvas. Essa classificação seguiu os trabalhos de Costa et al. (2002) e comunicação pessoal (Dra. Maria Elizabeth Fernandes Correia - EMBRAPA Agrobiologia). A comparação da riqueza de grupos entre as áreas foi feita utilizando-se análise de variância (teste F; $\mathrm{p} \leq 0,05$ ). 


\section{Resultados e Discussão}

Quanto à atividade dos grupos taxonômicos da fauna epígea, considerando o número de indivíduos por armadilha ao dia (Tabela 1), pode-se verificar que na área de plantio de café o grupo com maior atividade foi Isopoda, o que representou com 52,41 \% de indivíduos coletados; seguido do grupo Formicidae com 18,46\%. Considerando que o grupo Isopoda é um dos principais decompositores da matéria orgânica, esse resultado é positivo, em virtude da importância desse grupo no processo de ciclagem de nutrientes. A porcentagem de Isopoda foi considerada alta quando comparada com outros estudos (Moço et al., 2005; Silva et al., 2012). Moço et al. (2005) ao estudarem uma área de floresta preservada, verificaram valores de $8 \%$ e $15 \%$ do total de indivíduos coletados nos estratos serapilheira e solo, respectivamente. Silva et al. (2012), ao comparem a fauna do solo entre diferentes manejos de café, constataram atividade de Isopoda com variação entre 1,02 \% a 7,91\%. Já Tacca et al. (2017), ao estudarem bosque de eucalipto e remanescente de mata nativa, observaram valores de 1,91\% e 14,69\% do total de indivíduos coletados.

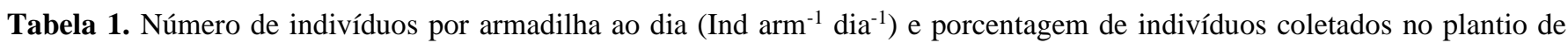
café (Coffea conephora) e plantio de eucalipto (Eucalyptus citriodora).

\begin{tabular}{ccccc}
\hline Grupos & \multicolumn{2}{c}{ Café } & \multicolumn{2}{c}{ Eucalipto } \\
& Ind arm $^{\mathbf{1}} \mathbf{d i a}^{\mathbf{- 1}}$ & $\mathbf{\%}$ & ${\text { Ind } \mathbf{~ a r m}^{\mathbf{- 1}} \mathbf{d i a}^{\mathbf{- 1}}}^{\mathbf{\%}}$ \\
\hline Araneae & 3,0 & 2,69 & 1,3 & 2,58 \\
Coleoptera & 3,3 & 2,95 & 0,3 & 0,60 \\
Collembola & 3,2 & 2,87 & 2,7 & 5,35 \\
Diptera & 4,1 & 3,67 & 1,4 & 2,78 \\
Formicidae & 20,6 & 18,46 & 42 & 83,34 \\
Isopoda & 58,5 & 52,41 & 1,7 & 3,36 \\
Isoptera & 7,3 & 6,55 & 0,2 & 0,40 \\
Larva de Coleoptera & 1,2 & 1,07 & 0,2 & 0,40 \\
Larva de Lepidoptera & 10,4 & 9,33 & 0,0 & 0,00 \\
Orthoptera & 0,0 & 0,00 & 0,5 & 0,99 \\
Thysanoptera & 0,0 & 0,00 & 0,1 & 0,2 \\
\hline Total & 111,6 & 100 & 50,4 & 100 \\
\hline
\end{tabular}

Fonte: Autores.

Para a área de plantio de eucalipto o grupo taxonômico com maior atividade foi Formicidae, perfazendo 83,34 \% de indivíduos coletados (Tabela 1). Esse grupo é comumente observado em elevada abundância em sistema naturais ou manejados. Caracterizando a fauna edáfica em diferentes coberturas vegetais na região norte fluminense, Moço et al. (2005), verificaram no povoamento de eucalipto, nos estratos serapilheira-solo, nas estações inverno e verão, valores entre 32 a $68 \%$ para o grupo Formicidae, e ainda altas frequências em áreas de capoeira e pasto; porém, na área de floresta não preservada, a frequência foi maior (74\%), sendo o valor mais próximo ao observado nesse estudo. O grupo Formicidae apresenta uma relação conhecida com o solo, em que na atividade de construção dos seus ninhos, promove o revolvimento do solo e incorporação da matéria orgânica utilizada como alimento (Vaz-de-Melo et al., 2009). Contudo, em grande abundância, pode representar um desequilíbrio, resultado da qualidade do alimento que favorece sua população, em detrimento aos demais grupos da fauna invertebrada. 
No que se refere aos valores totais de indivíduos coletados (Tabela 1), no plantio de café foi verificado mais que o dobro de indivíduos em comparação com a área com plantio de eucalipto. Uma possível explicação para o maior número de indivíduos coletados no plantio de café deve-se a serapilheira existente na área, que disponibiliza recursos alimentares, bem como a estrutura do micro-habitat gerado, possibilitando, dessa forma, melhores condições e recursos para o desenvolvimento da fauna como um todo.

Em estudos sobre a fauna edáfica sob diferentes manejos de café no Espírito Santo, estado que se destaca na produção dessa cultura, Teixeira et al. (2014) verificaram maior abundância na área de monocultura em relação ao consorcio de café com teca (Tectona grandis L. f.) e outro com cedro australiano (Toona ciliata M.). Avaliando a fauna invertebrada em cultivo de café com manejo convencional e orgânico, Silva et al. (2012) observaram maiores valores em comparação os quantificados nesse estudo. Já o menor número de organismos na área de eucalipto pode estar relacionado à qualidade do material aportado. De acordo com Camara et al. (2019), a serapilheira produzida por eucaliptos não favorece a biota edáfica decompositora, uma vez que esses organismos são caracterizados por apresentarem baixa qualidade nutricional, pois suas folhas possuem compostos químicos secundários que as tornam com baixa palatabilidade para os organismos decompositores (Chaer \& Tótola, 2007). No estudo de Hepp et al. (2009), que avaliaram a decomposição de espécies arbóreas, os autores observaram que a complexidade química das folhas de E. grandis pode ter influenciado em sua decomposição, em virtude de alguns compostos presentes, promovendo assim a atividade inibitória sobre organismos decompositores.

Os valores de riqueza total nas áreas de estudo foram muito próximos (Tabela 2). No entanto, pode-se observar diferença nos valores da riqueza média, sendo o maior valor verificado na área de plantio de café. $\mathrm{O}$ valor de riqueza na área de café foi próximo aos observados por Teixeira et al. (2014), em que os autores quantificaram valores de 7 e 10 de riqueza total no período chuvoso e seco, consecutivamente. Em estudo sobre a caracterização da fauna edáfica em diferentes coberturas vegetais, Moço et al. (2005) verificaram valores de riqueza média próximo ao encontrado neste estudo para a área de plantio de eucalipto. Esses autores observaram para a mesma área valores de ordem 4,4 e 5,6 para os estratos solo e seraplheira, respectivamente. Pode-se inferir, portanto, que a riqueza e atividade dos grupos está relacionada à qualidade da serapilheira aportada no solo, visto que ela representa o habitat da fauna epígea.

Tabela 2. Valores de riqueza total, riqueza média e índice de Shannon, índice de Pielou e índice de Simpson nas áreas de café (Coffea conephora) e eucalipto (Eucalyptus citriodora).

\begin{tabular}{cccccc}
\hline Áreas & Riqueza total & Riqueza media & Shannon & Pielou & Simpson \\
\hline Café & 9 & $6,40^{*}$ & 0,66 & 0,36 & 0,33 \\
Eucalipto & 10 & $4,60^{*}$ & 0,33 & 0,21 & 0,70 \\
\hline
\end{tabular}

*Diferença entre as áreas pelo teste de F a 5\% de probabilidade.

Fonte: Autores.

Os maiores valores de uniformidade e diversidade, índices de Pielou e Shannon, respectivamente, foram identificados na área de plantio de café (Tabela 2). Este fato possivelmente deve-se à distribuição e riqueza média de grupos nessa área que pode ter sido influenciada pelo manejo com adubação aliado à poda, que adicionou mais material vegetal ao solo, com melhor qualidade. A maior riqueza de grupos taxonômicos na área de café aliada à menor abundância de poucos grupos, contribuiu para uma maior uniformidade e diversidade nessa área em comparação com o povoamento de eucalipto. O valor de Shannon na área de café foi maior que os observados por Teixeira et al. (2014) em diferentes manejos de café, no município de Sooretama, ES. Contudo, esses autores verificaram maiores valores do índice de Pielou em relação a esse estudo. Na área de plantio de café os baixos valores do índice de Pielou podem ser explicados pelo manejo, visto que nesse estudo o material 
vegetal ficou depositado sobre o solo, e assim contribuiu para o maior número de indivíduos do grupo Isopoda, em detrimento a outros grupos, resultando no menor valor de uniformidade.

O maior valor de dominância (índice de Simpson) foi verificado na área com plantio de eucalipto, com valor de 0,70 (Tabela 2). A explicação para a maior dominância nessa área deve-se a maior abundância de indivíduos do grupo Formicidae. O valor observado para o índice de Simpson no presente estudo foi menor que o observado por Bueno et al. (2020) em áreas de plantio de eucalipto com seis e oito anos; porém, os autores constataram alta dominância de Acari.

Os grupos taxonômicos foram reunidos em sete grupos funcionais, conforme proposto por Costa (2002), a partir de características relativas ao uso do habitat e a principal forma de utilização do recurso alimentar (Tabela 3).

Tabela 3. Abundância e porcentagem de indivíduos coletados para os diferentes grupos funcionais, nas áreas de café (Coffea conephora) e eucalipto (Eucalyptus citriodora).

\begin{tabular}{lcccc}
\hline \multirow{2}{*}{ Grupos funcionais } & \multicolumn{2}{c}{ Café } & \multicolumn{2}{c}{ Eucalipto } \\
\cline { 2 - 5 } & $\begin{array}{c}\mathrm{N}^{\circ} \text { de indivíduos } \\
\text { coletados }\end{array}$ & $\%$ & $\begin{array}{c}\mathrm{N}^{\circ} \text { de indivíduos } \\
\text { coletados }\end{array}$ & $\%$ \\
\hline Social: Formicidae & 206 & 18,46 & 420 & 83,33 \\
Social: Isoptera & 73 & 6,54 & 2 & 0,40 \\
Holometabolos & 74 & 6,63 & 17 & 3,37 \\
Saprófagos & 585 & 52,52 & 23 & 4,56 \\
Predadores & 30 & 2,69 & 13 & 2,58 \\
Micrófagos & 32 & 2,87 & 27 & 5,36 \\
Larvas & 116 & 10,39 & 2 & 0,40 \\
Total & 1116 & 100 & 504 & 100 \\
\hline
\end{tabular}

Fonte: Autores.

$\mathrm{Na}$ área de plantio de café verificou-se maior percentagem do grupo funcional Saprófagos $(52,52 \%)$, seguido de Social: Formicidae com 18,46 \% (Tabela 3). O maior valor do grupo funcional Saprófago deve-se ao maior número de indivíduos coletados do grupo taxonômico Isopoda. Desse modo, a saprofagia representou a principal forma de alimentação na área de cultivo do café.

A presença do grupo de saprófago é um fator positivo para o sistema de cultivo de café, considerando que o grupo mais abundante - Isopoda - atua principalmente na fragmentação do material vegetal, sendo com isso, considerados decompositores primários (Correia et al., 2008). Neste sentido, a contribuição desse grupo no solo, está relacionada ao serviço ambiental de ciclagem de nutrientes (Brown et al., 2015). A macrofauna saprófaga, como Isopoda e outros grupos, fragmentam a serapilheira e contribuem para a decomposição, consumindo matéria em decomposição e movendo matéria orgânica ao longo do perfil do solo, ou seja, influenciam com isso na dinâmica do carbono no solo e sua taxa de decomposição (FAO, 2020). De acordo com Baretta et al. (2011), esses organismos são sensíveis a ambientes secos, em virtude da elevada taxa de evaporação; no entanto, possuem a capacidade de adaptação em ambientes diferentes.

Por outro lado, o grupo funcional Social: Formicidae, que foi o segundo grupo mais abundante na área de plantio de café, representou a maior pocentagem na área de eucalipto (83\%) (Tabela 3). A elevada abundância desses organismos pode estar relacionada à oferta de material vegetal no solo. Além disso, a ausência de grupos predadores nessa área, pode ter possibilitado o aumento populacional de formigas. As formigas são animais que dominam a maioria dos ecossistemas 
terrestres, sendo capazes de colonizar ambientes terrestres que oferecem poucos recursos para o desenvolvimento dos organismos edáficos (Vaz-de-Melo et al., 2009). O hábito de construção de galerias possui grande influência na capacidade de armazenamento e distribuição da água no solo (Baretta et al., 2011). Adicionalmente, esse grupo pode contribuir com o ciclo de nutrientes de pelo menos duas maneiras, na construção dos ninhos que geralmente envolvem a cominuição de animais vivos e material vegetal morto, e por representarem uma grande porcentagem dos itens alimentares para pequenos vertebrados (FAO, 2020).

\section{Conclusão}

O plantio de café favoreceu a atividade e diversidade da fauna, em que foi verificada maior abundância de indivíduos, riqueza de grupos e maior diversidade.

A análise da funcionalidade mostrou que o plantio de café proporcionou melhores condições para o grupo saprófago, representado pelo táxon Isopoda, enquanto na área de plantio de eucalipto houve predominância do grupo social Formicidae.

As sugestões são desenvolvimento de trabalhos em outros ambientes florestais nativos e plantados e com culturas perenes para confirmar os padrões utilizados.

\section{Referências}

Aquino, A. M., Aguiar-Menezes, E. de L., \& Queiroz, J. M. (2006) Recomendações para coleta de artrópodes terrestres por armadilhas de queda ("PitfallTraps"). Seropédica Embrapa Agrobiologia (Circular Técnica -INFOTECA-E).

Baretta, D., Santos, J. C. P., Segat, J. C., Geremia, E. V., Oliveira Filho, L. C., \& Alves, M. V. (2011) Fauna edáfica e qualidade do solo. Tópicos em Ciências do Solo, 7, 119-170.

Brito, M. F., Tsujigushi, B. P., Otsubo, A. A., Silva, R. F., \& Mercante, F. M. (2016) Diversidade da fauna edáfica e epigéica de invertebrados em consórcio de mandioca com adubos verdes. Pesquisa Agropecuária Brasileira, 51(3), 253-260.

Bueno, D., Silva, R. F., Almeida, H. S., Rodrigues, A. C., Vanzan, M., \& Andreazza, R. (2020) Influence of eucalyptus development under soil fauna. Brazilian Journal of Biology, 80(2), 345-353.

Brown, G. G., Niva, C. C., Zagatto, M. R. G., Ferreira, S. A., Nadolny, H. S., Cardoso, G. B. X., Santos, A., Martinez, G. A., Pasini, A., Bartz, M. L. C., Sautter, K. D., Thomazini, M. J., Baretta, D., Silva, E., Antoniolli, Z. I., Decaëns, T., Lavelle, P. M., Sousa, J. P., \& Carvalho, F. (2015) Biodiversidade da fauna do solo e sua contribuição para os serviços ambientais. In: Parron, L. M., Garcia, J. R., Oliveira, E. B., Brown, G. G., \& prado, R. B. (Ed.). Serviços ambientais em sistemas agrícolas e florestais do bioma Mata Atlântica. Brasília: Embrapa, 113-145.

Camara, R., Silva, V. D., Correia, M. E. F., \& Villela, D. M. (2019) Impacto da remoção de serapilheira sobre a comunidade de artrópodes edáficos em plantios abandonados de Corymbia citriodora. Ciência Florestal, 29(1), 14-26.

Casaril, C. E., Oliveira Filho, L. C. I., Santos, J. C. P., \& Rosa, M. G. (2019) Fauna edáfica em sistemas de produção de banana no Sul de Santa Catarina. Revista Brasileira de Ciências Agrárias, 14(1) e5613.

Chaer, G. M., \& Tótola, M. R. (2007) Impacto do Manejo de Resíduos Orgânicos durante a reforma de plantios de Eucalipto sobre indicadores de qualidade do solo. Revista Brasileira de Ciência do Solo, 31(6), 1381-1396.

Correia, M. E. F., Aquino, A. M., \& Aguirar-Menezes, E. L. (2008) Aspectos ecológicos dos isopoda terrestres. Seropédica Embrapa Agrobiologia, 23 p. (Documentos/ Embrapa Agrobiologia).

Costa, P. (2002) Fauna do solo em plantios experimentais de Eucalyptus grandis Maiden, Pseudosamanea guachapele Dugand e Acacia mangium Willd. 93p. Dissertação - Instituto de Agronomia, Universidade Federal Rural do Rio de Janeiro, Seropédica.

FAO., ITPS., GSBI., CBD., \& EC. (2020) State of knowledge of soil biodiversity - Status, challenges and potentialities, Report. Rome, FAO.

Food and Agriculture Organization of the United Nations - FAO (2015) Revised World Soil charter. Roma. 10p

Frouz, J. (2018) Effects of soil macro- and mesofauna on litter decomposition and soil organic matter stabilization. Geoderma. 332, 161-172.

Gallo, D., Nakano, O., Silveira Neto, S., Carvalho, R. P. L., Baptista, G. C. de., Berti Filho, E., Parra, J. R. P., Zuchi, R. A., Alves, S. B., \& Verdramim, J. D. (2002) Manual de entomologia agrícola. (2a ed.) Agronômica Ceres, 649p.

Hepp, L. U., Delanora, R., \& Trevisan, A. (2009) Compostos secundários durante a decomposição foliar de espécies arbóreas em um riacho do sul do Brasil. Acta Botânica Brasilica, 23(2), 407-413.

Lavelle, P. (1994) Faunal activities and soil processes: adaptive strategies that determine ecosystem function. In: XVth Congress, I, Acapulco, Mexico. 189220. 
Lavelle, P., \& Spain, A.V. (2001) Soil ecology. Dordrecht: Kluwer Academic. 654p

Marasas, M. E., Sarandón, S. J., \& Cicchino, A. C. (2001) Changes in soil arthropod functional group in a wheat crop under conventional and no tillage systems in Argentina. Applied Soil Ecology, 18(1), 61-68.

Moldenke, A. R. Arthropods (1994) In Weaver, R. W., Angle, S., Bottomley, P., Bezdicek, D., Smith, S., Tabatabai, A., Wollum, A. eds. Methods of soil analysis Microbial and biochemical properties. Part 2. Madison. SSSA, 517-542.

Moço, M. K., Gama-RodrigueS, E. F., Gama-Rodrigues, A. C., \& Correia, M. E. F. (2005) Caracterização da fauna edáfica em diferentes coberturas vegetais na região norte fluminense. Revista Brasileira de Ciência do Solo, 29, 555-564.

Odum, E. P. Ecologia (1986) Rio de Janeiro: Guanabara, 434p.

Oliveira, M. P. A., Bastos-Pereira, R., Torres, S. H. S., Pereira, T. S., Batista, F. M., Alves, J. P., Iniesta, L. F. M., Bouzan, R. S., Chagas-JR, A., Prous, X., Pietrobon, T., \& Ferreira, R. L. (2019) Choosing sampling methods for Chilopoda, Diplopoda and Isopoda (Oniscidea): A case study for ferruginous landscapes in Brazilian Amazonia. Applied Soil Ecology, 143, 181-191.

Oliveira, A. M., Barreto-Garcia, P. A. B., Novaes, A. B., carvalho, F. F., \& Meireles, I. E. S. (2020) Decomposição da serapilheira foliar em plantios de bambu, nim indiano e eucalipto. Ciência Florestal, 30(3), 845-855.

Pereira, G. H. A., Pereira, M. G., Anjos, L. H. C., Amorim, T. de A., \& Menezes, C. E. G. (2013) Decomposição da serrapilheira, diversidade e funcionalidade de invertebrados do solo em um fragmento de Floresta Atlântica. Bioscience Journal, 29(5), 1317-1327.

Sá, R. C., Pereira, M. G., \& Fontana, A. (2003) Características físicas e químicas de solos de tabuleiros em Sooretama (ES). Floresta e Ambiente, 10(2), 95 99, 2003.

Santos Neto, A. P., Barreto, P; A. B., Gama-Rodrigues, E. F., Novaes, A. B., \& Paula, A. (2015) Produção de serapilheira em floresta estacional semidecidual e em plantios de Pterogyne nitens Tul. e Eucalyptus urophylla S. T. Blake no sudoeste da Bahia. Ciência Florestal, 25(3), 633-643.

Silva, J., Jucksch, I., Maia, C. I., Feres, A., \& Tavares, R. C. (2012) Fauna do solo em sistemas de manejo com café. Journal of Biotechnology and Biodiversity, 3(2), 59-71.

Swift, M. J., Heal, O. W., \& Anderson, J. M. (1979) Decomposition in terrestrial ecosystems. Oxford, Blackwell, 372p.

Tacca, D., Klein, C., \& Preuss, J. F. (2017) Artropodofauna do solo em um bosque de eucalipto e um remanescente de mata nativa no sul do Brasil. Revista Thema, 14(2), 49-261.

Teixeira, A. F. R., Silva, V. M., \& Mendonça, E. S. (2014) Fauna edáfica em sistemas arborizados de café em solo de tabuleiros costeiros. Coffee Science, $9(3), 385-393$.

Vaz-de-Melo, F. V., Brown, G. G., Constantino, R., Louzada, J. N. C., Luizão, F. J., Morais, J. W., \& Zanetti, R. (2009) A importância da mesa e macrofauna do solo na fertilidade e como biondicadores. Biologia do solo, 38p. 